\title{
Convergent synthesis of the tetrasaccharide repeating unit of the cell wall lipopolysaccharide of Escherichia coli $\mathbf{0 4 0}$
}

\author{
Abhijit Sau and Anup Kumar Misra*
}

\author{
Full Research Paper \\ Address: \\ Bose Institute, Division of Molecular Medicine, P-1/12, C.I.T. Scheme \\ VII-M, Kolkata-700054, India; FAX: +91-33-2355 3886 \\ Email: \\ Anup Kumar Misra* - akmisra69@gmail.com \\ * Corresponding author \\ Keywords: \\ Escherichia coli; glycosylation; lipopolysaccharide; O-antigen; \\ tetrasaccharide
}

\author{
Beilstein J. Org. Chem. 2012, 8, 2053-2059. \\ doi:10.3762/bjoc. 8.230 \\ Received: 30 August 2012 \\ Accepted: 01 November 2012 \\ Published: 22 November 2012 \\ Associate Editor: S. Flitsch \\ (c) 2012 Sau and Misra; licensee Beilstein-Institut. \\ License and terms: see end of document.
}

\begin{abstract}
A tetrasaccharide repeating unit corresponding to the cell-wall lipopolysaccharide of E. coli O40 was synthesized by using a convergent block glycosylation strategy. A disaccharide donor was coupled to a disaccharide acceptor by a stereoselective glycosylation. A 2-aminoethyl linker was chosen as the anomeric protecting group at the reducing end of the tetrasaccharide. All glycosylation steps are significantly high yielding and stereoselective.
\end{abstract}

\section{Introduction}

Infantile diarrhoea is one of the major causes of morbidity and mortality in infancy in developing countries [1]. Among several factors, Escherichia coli (E. coli) infection is one of the major causes of diarrhoeal disease in the developing countries [2]. E. coli are Gram-negative opportunistic pathogens and belong to the genus Enterobacteriaceae. In general, E. coli is considered as a friendly organism present in the normal intestinal flora of humans and animals and can kill harmful bacteria by producing vitamins and other immunostimulants [3]. However, a number of $E$. coli strains acquire virulence factors and cause severe intestinal and urinary-tract infections [4,5]. E. coli serotypes are generally classified based on the somatic, flagella and capsular antigens [6]. Diarrhoea-causing E. coli strains are broadly classified in four categories: (a) Enteropathogenic E. coli infects through the production of heat-labile and heatstable toxins; (b) enteroinvasive $E$. coli acts through the invasion of the host body; (c) enteropathogenic E. coli infects by adhering to the membrane of the host intestine; and (d) verotoxin $E$. coli infects by the production of verotoxin or shiga toxin [7]. Recently, Zhao et al. reported the structure of the repeating unit of the cell-wall antigenic lipopolysaccharide of E. coli $\mathrm{O} 40$ [8], which contains two D-galactosyl moieties with alpha and beta linkage, one beta-linked D-glucosamine and one beta-linked D-mannosyl moiety (Figure 1). 


$$
\rightarrow 4)-\alpha-D-G a l p-(1 \rightarrow 3)-\beta-D-G l c p N A c-(1 \rightarrow 2)-\beta-D-G a l p-(1 \rightarrow 4)-\beta-D-M a n p-(1 \rightarrow
$$

Figure 1: Structure of the tetrasaccharide repeating unit of the cell-wall lipopolysaccharide of Escherichia coli O40.

Although several therapeutics have appeared in the past to control the diarrheal epidemics caused by E. coli infections, emergence of resistant strains is a serious concern in the development of therapeutics against this organism. Since, bacterial cell-wall lipopolysaccharides play important roles in the pathogenicity of the virulent strains, it would be pertinent to develop glycoconjugate therapeutics based on the cell-wall oligosaccharide haptens to reduce the number of infections [9-12]. In order to evaluate the therapeutic efficacy of the glycoconjugate derivatives it is essential to have a significant quantity of oligosaccharides, which is difficult to isolate from natural sources. Therefore, the development of a chemical synthetic strategy for the synthesis of the oligosaccharides and their close analogues can add momentum towards the preparation of glycoconjugatebased therapeutics. In this perspective, we report herein a concise chemical synthesis of the tetrasaccharide repeating unit of the cell-wall lipopolysaccharide of E. coli O40, using a convergent block synthetic strategy.

\section{Results and Discussion}

The target tetrasaccharide $\mathbf{1}$ as its 2-aminoethyl glycoside was synthesized by a stereoselective glycosylation of a disaccharide acceptor $\mathbf{8}$ and a disaccharide thioglycoside donor $\mathbf{9}$ using a $[2+2]$ block synthetic strategy. The disaccharide intermediates were synthesized from the suitably protected monosaccharide derivatives 2 [13], 3 [14], 4 [15] and 5 [16], which were prepared from the commercially available reducing sugars, by applying a series of functional group protection-deprotection methodologies (Figure 2). The synthetic strategy has a number of notable features, which include (a) stereoselective [2 +2$]$ block glycosylation; (b) application of general glycosylation reactions by using thioglycosides as glycosyl donors and a combination of $\mathrm{N}$-iodosuccinimide (NIS) and perchloric acid supported over silica $\left(\mathrm{HClO}_{4}-\mathrm{SiO}_{2}\right)[17,18]$ as glycosyl activator; (c) exploitation of the armed-disarmed glycosylation concept for the orthogonal activation of thioglycoside during the synthesis of disaccharide derivative 9 [19]; (d) use of aminoethyl linker as the anomeric protecting group; (e) removal of benzyl groups using a combination of triethylsilane and $\mathrm{Pd}(\mathrm{OH})_{2}-\mathrm{C}[20]$; and (f) preparation of $\beta$-D-mannosidic moiety from the $\beta$-D-glucoside [13].

Benzylation of 2-azidoethyl 3-O-benzyl-4,6- $O$-benzylidene- $\beta$ D-mannopyranoside (2) [13] (prepared from D-glucose in nine steps) by using benzyl bromide and sodium hydroxide [21] followed by reductive ring opening of the 4,6-O-benzylidene acetal with triethylsilane and iodine [22] furnished compound 6 in $82 \%$ yield. Stereoselective glycosylation of compound 6 with thioglycoside derivative 3 in the presence of a combination of $N$-iodosuccinimide (NIS) and $\mathrm{HClO}_{4}-\mathrm{SiO}_{2}$ [17] gave disaccharide derivative 7 in a $77 \%$ yield. Formation of compound 7 was confirmed from its spectral analysis [signals at $\delta 4.73(\mathrm{~d}, J=$ $\left.8.0 \mathrm{~Hz}, \mathrm{H}-1_{\mathrm{B}}\right), 4.41$ (br s, $\left.\mathrm{H}-1_{\mathrm{A}}\right)$ in the ${ }^{1} \mathrm{H}$ NMR and at $\delta 101.6$ $\left(\mathrm{C}-1_{\mathrm{A}}\right), 100.7\left(\mathrm{C}-1_{\mathrm{B}}\right)$ in the ${ }^{13} \mathrm{C}$ NMR spectra respectively]. Saponification of compound 7 by using sodium methoxide followed by 3,4-O-isopropylidenation with 2,2-dimethoxypropane and $p$-toluenesulfonic acid [23] furnished disaccharide derivative $\mathbf{8}$ in $74 \%$ yield (Scheme 1).

In a separate experiment, stereoselective glycosylation of thioglycoside derivative $\mathbf{4}$ with the thioglycoside acceptor $\mathbf{5}$ in the presence of a combination of NIS and $\mathrm{HClO}_{4}-\mathrm{SiO}_{2}$ [17] in dichloromethane-diethyl ether furnished disaccharide thioglycoside derivative 9 in a $74 \%$ yield together with a minor quantity of its other isomer $(\approx 5 \%)$, which was separated by column chromatography. Formation of compound 9 was confirmed from its spectral analysis $\left[\delta 5.51\left(\mathrm{~d}, J=3.5 \mathrm{~Hz}, \mathrm{H}-1_{\mathrm{D}}\right), 5.37(\mathrm{~d}\right.$, $\left.J=10.5 \mathrm{~Hz}, \mathrm{H}-1_{\mathrm{C}}\right)$ in the ${ }^{1} \mathrm{H} \mathrm{NMR}$ and $\delta 97.4\left(\mathrm{C}-1_{\mathrm{D}}\right), 83.0$ $\left(\mathrm{C}-1_{\mathrm{C}}\right)$ in the ${ }^{13} \mathrm{C}$ NMR spectra, respectively]. During the synthesis of compound $\mathbf{9}$, thioglycoside $\mathbf{4}$ acted as glycosyl donor
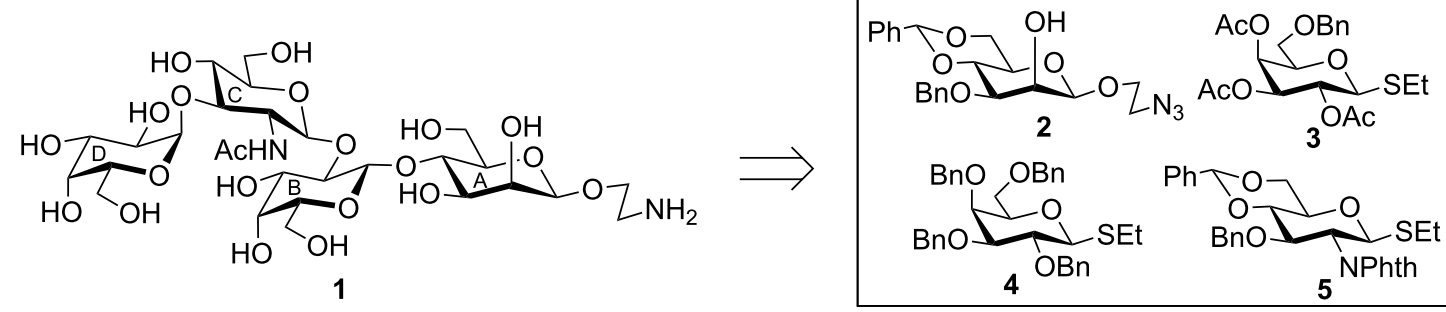

Figure 2: Structure of the synthesized tetrasaccharide 1 and its synthetic precursors. 


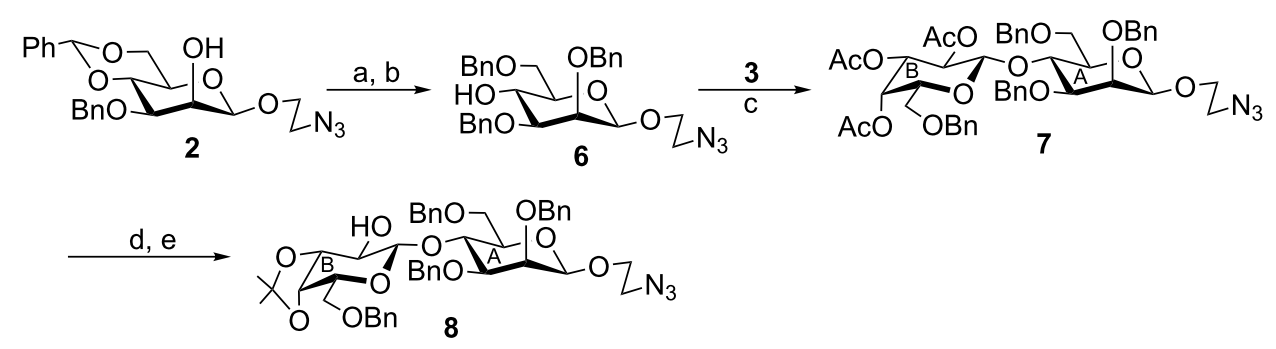

Scheme 1: Synthesis of disaccharide derivative 8. Reagents and conditions: (a) benzyl bromide, $\mathrm{NaOH}, \mathrm{DMF}$, room temperature, $1 \mathrm{~h}$; (b) Et ${ }_{3} \mathrm{SiH}, \mathrm{I}_{2}$, $\mathrm{CH}_{3} \mathrm{CN}, 0{ }^{\circ} \mathrm{C}, 1 \mathrm{~h}, 82 \%$; (c) NIS, $\mathrm{HClO}_{4}-\mathrm{SiO}_{2}, \mathrm{MS} 4 \AA, \mathrm{CH}_{2} \mathrm{Cl}_{2},-25^{\circ} \mathrm{C}, 1 \mathrm{~h}, 77 \%$; (d) $0.1 \mathrm{M} \mathrm{CH}_{3} \mathrm{ONa}, \mathrm{CH} \mathrm{H}_{3} \mathrm{OH}$, room temperature, $3 \mathrm{~h}$; (e) $2,2-$ dimethoxypropane, $p$-TsOH, DMF, room temperature, $5 \mathrm{~h}, 74 \%$.

and thioglycoside $\mathbf{5}$ acted as orthogonal glycosyl acceptor because of the difference in their reactivity following the "armed-disarmed glycosylation" concept [19,24] (Scheme 2).

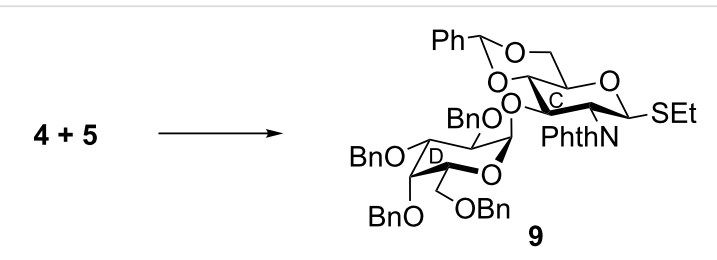

Scheme 2: Synthesis of disaccharide derivative 9. Reagents and conditions: (a) $\mathrm{NIS}, \mathrm{HClO}_{4}-\mathrm{SiO}_{2}, \mathrm{MS} 4 \AA, \mathrm{CH}_{2} \mathrm{Cl}_{2}-\mathrm{Et}_{2} \mathrm{O},-25^{\circ} \mathrm{C}, 1 \mathrm{~h}$, $74 \%$.

Iodonium ion promoted [2+2] stereoselective glycosylation of compound 8 and compound 9 in the presence of NIS and $\mathrm{HClO}_{4}-\mathrm{SiO}_{2}$ [17] furnished tetrasaccharide derivative $\mathbf{1 0}$ in $71 \%$ yield. Formation of compound $\mathbf{1 0}$ was confirmed by its spectral analysis [signals at $\delta 101.6\left(\mathrm{C}-1_{\mathrm{B}}\right), 101.5(\mathrm{PhCH})$, $100.8\left(\mathrm{C}-1_{\mathrm{C}}\right), 100.2\left(\mathrm{C}-1_{\mathrm{A}}\right), 97.3\left(\mathrm{C}-1_{\mathrm{D}}\right)$ in the ${ }^{13} \mathrm{C}$ NMR spectrum]. Compound $\mathbf{1 0}$ was subjected to a sequence of reactions involving (a) removal of $N$-phthalimido group by using hydrazine hydrate [25]; (b) $\mathrm{N}$-acetylation by using acetic anhydride and pyridine; (c) removal of isopropylidene ketal and benzylidene acetal by acid hydrolysis; and finally (d) removal of benzyl ethers by using triethylsilane and $20 \% \mathrm{Pd}(\mathrm{OH})_{2}-\mathrm{C}$
[20] to furnish target compound 1, which was purified through a Sephadex ${ }^{\circledR}$ LH-20 column to give pure compound 1 in $60 \%$ overall yield. Spectral data of compound 1 confirmed its formation [signals at $\delta 5.31\left(\mathrm{~d}, J=8.5 \mathrm{~Hz}, \mathrm{H}-1_{\mathrm{C}}\right), 5.15(\mathrm{~d}, J=3.5 \mathrm{~Hz}$, $\left.\mathrm{H}-1_{\mathrm{D}}\right), 4.63($ br s, H-1 $\mathrm{A}), 4.34\left(\mathrm{~d}, J=8.5 \mathrm{~Hz}, \mathrm{H}-1_{\mathrm{B}}\right)$ in the ${ }^{1} \mathrm{H}$ NMR and at $\delta 100.7\left(\mathrm{C}-1_{\mathrm{B}}\right), 100.6\left(\mathrm{C}-1_{\mathrm{B}}\right), 99.6\left(2 \mathrm{C}, \mathrm{C}-1_{\mathrm{A}}\right.$, $\mathrm{C}-1_{\mathrm{C}}$ ) in the ${ }^{13} \mathrm{C}$ NMR] (Scheme 3 ).

\section{Conclusion}

In summary, synthesis of a tetrasaccharide repeating unit corresponding to the cell-wall lipopolysaccharide of E. coli $\mathrm{O} 40$ was achieved by using a convergent $[2+2]$ block synthetic strategy. The yields are excellent in all reactions. A general reaction condition was used in all glycosylation reactions. All intermediates and final compounds were characterized by their spectral analysis. The armed-disarmed glycosylation concept was applied for the synthesis of disaccharide derivative 9. A 2-Aminoethyl linker was used as the anomeric protecting group.

\section{Experimental}

General methods: All reactions were monitored by thin-layer chromatography over silica-gel-coated TLC plates. The spots on TLC were visualized by warming ceric sulfate $\left(2 \% \mathrm{Ce}\left(\mathrm{SO}_{4}\right)_{2}\right.$ in $2 \mathrm{~N} \mathrm{H}_{2} \mathrm{SO}_{4}$ )-sprayed plates on a hot plate. Silica gel 230-400 mesh was used for column chromatography. ${ }^{1} \mathrm{H}$ and ${ }^{13} \mathrm{C}$ NMR spectra were recorded on Brucker Avance $500 \mathrm{MHz}$ by using

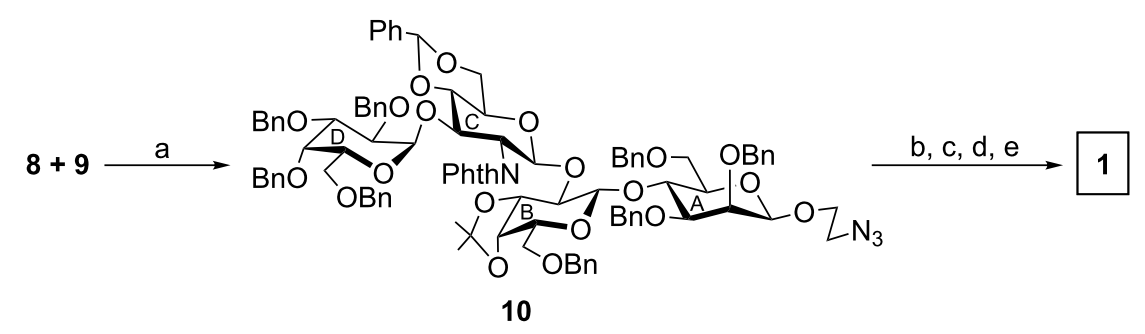

Scheme 3: Synthesis of target tetrasaccharide 1. Reagents and conditions: (a) $\mathrm{NIS}, \mathrm{HClO}_{4}-\mathrm{SiO}_{2}, \mathrm{CH}_{2} \mathrm{Cl}_{2},-15{ }^{\circ} \mathrm{C}, 1 \mathrm{~h}, 71 \%$; (b) $\mathrm{NH}_{2} \mathrm{NH}_{2} \cdot \mathrm{H}_{2} \mathrm{O}, \mathrm{EtOH}$, $90{ }^{\circ} \mathrm{C}, 5 \mathrm{~h}$; (c) acetic anhydride, pyridine, room temperature, $2 \mathrm{~h}$; (d) $80 \%$ aq $\mathrm{AcOH}, 80{ }^{\circ} \mathrm{C}, 1.5 \mathrm{~h} ;(\mathrm{e})$ triethylsilane, $20 \% \mathrm{Pd}(\mathrm{OH})_{2}-\mathrm{C}, \mathrm{CH} \mathrm{H}_{3} \mathrm{OH}, 6 \mathrm{~h}$, room temperature, overall $60 \%$. 
$\mathrm{CDCl}_{3}$ as solvent and TMS as internal reference, unless stated otherwise. Chemical shift values are expressed in $\delta \mathrm{ppm}$. MALDI-MS were recorded on a Bruker Daltronics mass spectrometer. Commercially available grades of organic solvents of adequate purity were used in all reactions. $\mathrm{HClO}_{4}-\mathrm{SiO}_{2}$ was prepared following the method reported in the literature [18].

2-Azidoethyl 2,3,6-tri- $O$-benzyl- $\beta$-D-mannopyranoside (6): To a solution of compound $2(2.0 \mathrm{~g}, 4.68 \mathrm{mmol})$ in dry DMF $(10 \mathrm{~mL})$ were added benzyl bromide $(1.2 \mathrm{~mL}, 10.09 \mathrm{mmol})$ and powdered $\mathrm{NaOH}(750.0 \mathrm{mg}, 18.75 \mathrm{mmol})$ and the reaction mixture was stirred at room temperature for $1 \mathrm{~h}$. The reaction mixture was diluted with water $(100 \mathrm{~mL})$ and extracted with $\mathrm{CH}_{2} \mathrm{Cl}_{2}(100 \mathrm{~mL})$. The organic layer was washed with $\mathrm{H}_{2} \mathrm{O}$, dried $\left(\mathrm{Na}_{2} \mathrm{SO}_{4}\right)$ and concentrated. The crude product was passed through a short pad of $\mathrm{SiO}_{2}$ by using hexane-EtOAc $(5: 1)$ as eluant to give the $O$-benzylated product $(2.2 \mathrm{~g}, 91 \%)$. A solution of the $O$-benzylated product $(2.2 \mathrm{~g}, 4.25 \mathrm{mmol})$ in dry $\mathrm{CH}_{3} \mathrm{CN}(20 \mathrm{~mL})$ was cooled to $0{ }^{\circ} \mathrm{C}$. To the cooled reaction mixture were added $\mathrm{Et}_{3} \mathrm{SiH}(1.4 \mathrm{~mL}, 8.76 \mathrm{mmol})$ and $\mathrm{I}_{2}$ (250.0 $\mathrm{mg}, 0.98 \mathrm{mmol}$ ), and the reaction mixture was stirred at the same temperature for $1 \mathrm{~h}$. The reaction mixture was diluted with $\mathrm{CH}_{2} \mathrm{Cl}_{2}(100 \mathrm{~mL})$ and the organic layer was successively washed with saturated $\mathrm{NaHCO}_{3}$ and $\mathrm{H}_{2} \mathrm{O}$, and then dried $\left(\mathrm{Na}_{2} \mathrm{SO}_{4}\right)$ and concentrated. The crude product was purified over $\mathrm{SiO}_{2}$ by using hexane-EtOAc (4:1) as eluant to give pure compound 6 (1.7 g, overall 82\%). White solid; mp 89-90 ${ }^{\circ} \mathrm{C}$; $[\alpha]_{\mathrm{D}}{ }^{25}-97\left(c\right.$ 1.0, $\left.\mathrm{CHCl}_{3}\right)$; IR (KBr): 3293, 2845, 2110, 1497, 1454, 1365, 1310, 1119, 1065, 779, 659, $599 \mathrm{~cm}^{-1} ;{ }^{1} \mathrm{H}$ NMR $\left(500 \mathrm{MHz}, \mathrm{CDCl}_{3}\right) \delta 7.43-7.23(\mathrm{~m}, 15 \mathrm{H}, \mathrm{Ar}-\mathrm{H}), 4.98(\mathrm{~d}, J=$ $\left.12.5 \mathrm{~Hz}, 1 \mathrm{H}, \mathrm{PhCH}_{2}\right), 4.75$ (d, $\left.J=12.5 \mathrm{~Hz}, 1 \mathrm{H}, \mathrm{PhCH}_{2}\right), 4.61$, $4.58\left(2 \mathrm{~d}, J=12.0 \mathrm{~Hz}, 2 \mathrm{H}, \mathrm{PhCH}_{2}\right.$ ), 4.48 (br s, 1H, H-1), 4.45 $\left(\mathrm{d}, J=12.0 \mathrm{~Hz}, 1 \mathrm{H}, \mathrm{PhCH}_{2}\right), 4.32\left(\mathrm{~d}, J=12.0 \mathrm{~Hz}, 1 \mathrm{H}, \mathrm{PhCH}_{2}\right)$, 4.15-4.11 (m, 1H,-OCH $2^{-}$), 3.96 (br s, 1H, H-2), 3.94 (t, $J=9.5$ $\mathrm{Hz}$ each, 1H, H-4), 3.85 (dd, $J=10.5,3.5 \mathrm{~Hz}, 1 \mathrm{H}, \mathrm{H}-6_{\mathrm{a}}$ ), 3.75 (dd, $\left.J=10.5,6.5 \mathrm{~Hz}, 1 \mathrm{H}, \mathrm{H}-6_{\mathrm{b}}\right), 3.66-3.62\left(\mathrm{~m}, 1 \mathrm{H}, \mathrm{OCH}_{2^{-}}\right)$, 3.58-3.53 (m, 1H, $\left.\mathrm{CH}_{2} \mathrm{~N}_{3}\right), 3.46-3.42(\mathrm{~m}, 1 \mathrm{H}, \mathrm{H}-5), 3.34-3.30$ $\left(\mathrm{m}, 1 \mathrm{H}, \mathrm{CH}_{2} \mathrm{~N}_{3}\right), 3.29(\mathrm{dd}, J=10.0,3.5 \mathrm{~Hz}, 1 \mathrm{H}, \mathrm{H}-3)$; ${ }^{13} \mathrm{C}$ NMR (125 MHz, $\left.\mathrm{CDCl}_{3}\right) \delta 138.6-127.4$ (Ar-C), 101.8 (C-1), 81.3 (C-3), 75.4 (C-5), $74.4\left(\mathrm{PhCH}_{2}\right), 73.7\left(\mathrm{PhCH}_{2}\right), 73.5$ (C-5), $71.1\left(\mathrm{PhCH}_{2}\right), 70.7$ (C-6), $68.6\left(\mathrm{OCH}_{2}\right), 68.0(\mathrm{C}-2), 50.9$ $\left(\mathrm{CH}_{2} \mathrm{~N}_{3}\right)$; ESI-MS: $542.2[\mathrm{M}+\mathrm{Na}]^{+}$; Anal. calcd for $\mathrm{C}_{29} \mathrm{H}_{33} \mathrm{~N}_{3} \mathrm{O}_{6}$ : C, 67.04; H, 6.40; found: C, 66.90; H, 6.58.

2-Azidoethyl $O$-(2,3,4-tri- $O$-acetyl-6- $O$-benzyl- $\beta$-Dgalactopyranosyl)-( $\rightarrow 4)-2,3,6$-tri- $O$-benzyl- $\beta$-D-mannopyranoside (7): To a solution of compound 3 (1.4 g, $3.18 \mathrm{mmol})$ and compound $6(1.5 \mathrm{~g}, 2.88 \mathrm{mmol})$ in anhydrous $\mathrm{CH}_{2} \mathrm{Cl}_{2}(10 \mathrm{~mL})$ was added MS $4 \AA(2.0 \mathrm{~g})$, and the reaction mixture was stirred at room temperature for $30 \mathrm{~min}$ under argon. The reaction mixture was cooled to $-25{ }^{\circ} \mathrm{C}$, and $N$-iodosuccinimide (NIS; $0.8 \mathrm{~g}, 3.55 \mathrm{mmol}$ ) and $\mathrm{HClO}_{4}-\mathrm{SiO}_{2}$ $(25.0 \mathrm{mg}$ ) were added to it. After being stirred at same temperature for $1 \mathrm{~h}$ the reaction mixture was filtered through a Celite ${ }^{\circledR}$ bed and washed with $\mathrm{CH}_{2} \mathrm{Cl}_{2}(100 \mathrm{~mL})$. The organic layer was successively washed with $5 \% \mathrm{Na}_{2} \mathrm{~S}_{2} \mathrm{O}_{3}$, saturated $\mathrm{NaHCO}_{3}$ and water, and then dried $\left(\mathrm{Na}_{2} \mathrm{SO}_{4}\right)$ and concentrated under reduced pressure to give the crude product. The crude product was purified over $\mathrm{SiO}_{2}$ by using hexane-EtOAc $(7: 1)$ as eluant to give pure compound 7 (2.0 g, 77\%). Yellow oil; $[\alpha]_{\mathrm{D}}{ }^{25}-13$ (c 1.0, $\mathrm{CHCl}_{3}$ ); IR (neat): 3087, 2956, 2153, 1605, 1487, 1345, 1254 , 1183, 1142, 1045, 999, 774, 734, 647, $542 \mathrm{~cm}^{-1}$; ${ }^{1} \mathrm{H}$ NMR (500 $\left.\mathrm{MHz}, \mathrm{CDCl}_{3}\right) \delta 7.35-7.16(\mathrm{~m}, 20 \mathrm{H}, \mathrm{Ar}-\mathrm{H}), 5.33(\mathrm{~d}, J=3.0 \mathrm{~Hz}$, $\left.1 \mathrm{H}, \mathrm{H}-4_{\mathrm{B}}\right), 5.06$ (t, $J=8.0 \mathrm{~Hz}$ each, $\left.1 \mathrm{H}, \mathrm{H}-2_{\mathrm{B}}\right), 4.87$ (d, $J=12.0$ $\left.\mathrm{Hz}, 1 \mathrm{H}, \mathrm{PhCH}_{2}\right), 4.84$ (dd, $\left.J=10.5,3.5 \mathrm{~Hz}, 1 \mathrm{H}, \mathrm{H}-3_{\mathrm{B}}\right), 4.73$ (d, $\left.J=8.0 \mathrm{~Hz}, 1 \mathrm{H}, \mathrm{H}-1_{\mathrm{B}}\right), 4.72-4.71(2 \mathrm{~d}, J=12.0 \mathrm{~Hz}$ each, $2 \mathrm{H}$, $\left.\mathrm{PhCH}_{2}\right), 4.59$ (d, $\left.J=12.0 \mathrm{~Hz}, 1 \mathrm{H}, \mathrm{PhCH}_{2}\right), 4.47,4.45$ (2 d, $J=$ $12.0 \mathrm{~Hz}$ each, $\left.2 \mathrm{H}, \mathrm{PhCH}_{2}\right), 4.41\left(\mathrm{br} \mathrm{s}, 1 \mathrm{H}, \mathrm{H}-1_{\mathrm{A}}\right), 4.39(\mathrm{~d}, J=$ $\left.12.0 \mathrm{~Hz}, 1 \mathrm{H}, \mathrm{PhCH}_{2}\right), 4.19$ (d, $\left.J=12.0 \mathrm{~Hz}, 1 \mathrm{H}, \mathrm{PhCH}_{2}\right), 4.14$ $\left(\mathrm{t}, J=9.0 \mathrm{~Hz}\right.$ each, $\left.1 \mathrm{H}, \mathrm{H}-4_{\mathrm{A}}\right), 4.10-4.05\left(\mathrm{~m}, 1 \mathrm{H}, \mathrm{OCH}_{2}\right), 3.92$ $\left(\mathrm{d}, J=3.0 \mathrm{~Hz}, 1 \mathrm{H}, \mathrm{H}-2_{\mathrm{A}}\right), 3.76-3.70\left(\mathrm{~m}, 2 \mathrm{H}, \mathrm{H}-6_{\mathrm{abA}}\right)$, 3.65-3.60 (m, $\left.1 \mathrm{H}, \mathrm{OCH}_{2}\right), 3.54-3.49\left(\mathrm{~m}, 2 \mathrm{H}, \mathrm{H}-5_{\mathrm{B}}, \mathrm{CH}_{2} \mathrm{~N}_{3}\right)$, 3.47 (dd, $\left.J=10.0,3.0 \mathrm{~Hz}, 1 \mathrm{H}, \mathrm{H}-3_{\mathrm{A}}\right), 3.42-3.38\left(\mathrm{~m}, 1 \mathrm{H}, \mathrm{H}-5_{\mathrm{A}}\right)$, $3.32-3.22\left(\mathrm{~m}, 3 \mathrm{H}, \mathrm{H}-6_{\mathrm{abB}}, \mathrm{CH}_{2} \mathrm{~N}_{3}\right), 1.99,1.95,1.90(3 \mathrm{~s}, 9 \mathrm{H}, 3$ $\left.\mathrm{COCH}_{3}\right) ;{ }^{13} \mathrm{C} \mathrm{NMR}\left(125 \mathrm{MHz}, \mathrm{CDCl}_{3}\right) \delta 169.9,169.8,169.4$ $\left(3 \mathrm{COCH}_{3}\right), 138.5-126.8(\mathrm{Ar}-\mathrm{C}), 101.6\left(\mathrm{C}-1_{\mathrm{A}}\right), 100.7\left(\mathrm{C}-1_{\mathrm{B}}\right)$, $80.6\left(\mathrm{C}-5_{\mathrm{B}}\right), 75.7\left(\mathrm{C}-5_{\mathrm{A}}\right), 74.7\left(\mathrm{C}-2_{\mathrm{A}}\right), 74.3\left(2 \mathrm{C}, \mathrm{C}-4_{\mathrm{A}}\right.$, $\mathrm{PhCH} 2), 73.7\left(\mathrm{PhCH}_{2}\right), 73.3\left(\mathrm{PhCH}_{2}\right), 71.7\left(\mathrm{C}-5_{\mathrm{B}}\right), 71.5$ $\left(\mathrm{PhCH}_{2}\right), 71.3\left(\mathrm{C}-3_{\mathrm{B}}\right), 70.0\left(\mathrm{C}-2_{\mathrm{B}}\right), 68.6\left(\mathrm{C}-6_{\mathrm{A}}\right), 68.5\left(\mathrm{OCH}_{2}\right)$, $67.4\left(\mathrm{C}-4_{\mathrm{B}}\right), 66.9\left(\mathrm{C}-6_{\mathrm{B}}\right), 50.8\left(\mathrm{CH}_{2} \mathrm{~N}_{3}\right), 20.7,20.6,20.5$ $\left(\mathrm{COCH}_{3}\right)$; MALDI-MS: $920.3[\mathrm{M}+\mathrm{Na}]^{+}$; Anal. calcd for $\mathrm{C}_{48} \mathrm{H}_{55} \mathrm{~N}_{3} \mathrm{O}_{14}$ : C, 64.20; H, 6.17; found: C, 64.06; H, 6.35.

2-Azidoethyl $O$-(6- $O$-benzyl-3,4- $O$-isopropylidene- $\beta$-Dgalactopyranosyl)-(1 $\rightarrow 4)-2,3,6$-tri- $O$-benzyl- $\beta$-D-mannopyranoside (8): A solution of compound $7(1.8 \mathrm{~g}, 2.0 \mathrm{mmol})$ in $0.1 \mathrm{M} \mathrm{CH}_{3} \mathrm{ONa}(25 \mathrm{~mL})$ was stirred at room temperature for $2 \mathrm{~h}$. The reaction mixture was neutralized with Dowex 50W X8 $\left(\mathrm{H}^{+}\right)$resin, filtered and concentrated. To a solution of the de- $O-$ acetylated product in dry DMF $(10 \mathrm{~mL})$ was added 2,2dimethoxypropane $(0.7 \mathrm{~mL}, 5.69 \mathrm{mmol})$ followed by $p$-TsOH $(0.2 \mathrm{~g})$ and the reaction mixture was stirred at room temperature for $5 \mathrm{~h}$. The reaction was quenched with $\mathrm{Et}_{3} \mathrm{~N}(1 \mathrm{~mL})$, the solvents were removed under reduced pressure, and the crude reaction mixture was diluted with $\mathrm{CH}_{2} \mathrm{Cl}_{2}(100 \mathrm{~mL})$. The organic layer was washed with saturated $\mathrm{NaHCO}_{3}$, dried $\left(\mathrm{Na}_{2} \mathrm{SO}_{4}\right)$ and concentrated to give the crude product, which was purified over $\mathrm{SiO}_{2}$ by using hexane-EtOAc (2:1) as eluant to give pure compound $8(1.2 \mathrm{~g}, 74 \%)$. Yellow oil; $[\alpha]_{\mathrm{D}}{ }^{25}-21$ (c 1.0, $\mathrm{CHCl}_{3}$ ); IR (neat): 3418, 3030, 2926, 2198, 1743, 1711, 1646, 1390, 1253, 1099, 1053, 864, 754, 667, $531 \mathrm{~cm}^{-1}$; 
${ }^{1} \mathrm{H}$ NMR (500 MHz, $\mathrm{CDCl}_{3}$ ) $\delta$ 7.30-7.14 (m, 20H, Ar-H), 4.84 $\left(\mathrm{d}, J=12.5 \mathrm{~Hz}, 1 \mathrm{H}, \mathrm{PhCH}_{2}\right), 4.63-4.40\left(\mathrm{~m}, 6 \mathrm{H}, \mathrm{PhCH}_{2}\right), 4.38$ $\left(\mathrm{d}, J=8.0 \mathrm{~Hz}, 1 \mathrm{H}, \mathrm{H}-1_{\mathrm{B}}\right), 4.33\left(\right.$ br s, $\left.1 \mathrm{H}, \mathrm{H}-1_{\mathrm{A}}\right), 4.30$ (d, $J=$ $\left.12.5 \mathrm{~Hz}, 1 \mathrm{H}, \mathrm{PhCH} H_{2}\right), 4.22$ (t, $J=9.5 \mathrm{~Hz}$ each, $\left.1 \mathrm{H}, \mathrm{H}-4_{\mathrm{A}}\right)$, $4.02-3.98\left(\mathrm{~m}, 2 \mathrm{H}, \mathrm{H}-2_{\mathrm{A}}, \mathrm{OCH}_{2}\right), 3.91(\mathrm{dd}, J=10.0,3.5 \mathrm{~Hz}$, $\left.\mathrm{H}-3_{\mathrm{B}}\right), 3.83$ (dd, $\left.J=12.0,5.5 \mathrm{~Hz}, 1 \mathrm{H}, \mathrm{H}-6_{\mathrm{aB}}\right), 3.81$ (d, $J=2.0$ $\left.\mathrm{Hz}, 1 \mathrm{H}, \mathrm{H}-4_{\mathrm{B}}\right), 3.76\left(\mathrm{dd}, J=12.0,2.0, \mathrm{~Hz}, 1 \mathrm{H}, \mathrm{H}-6_{\mathrm{bB}}\right), 3.71(\mathrm{br}$ $\left.\mathrm{s}, 1 \mathrm{H}, \mathrm{H}-5_{\mathrm{B}}\right), 3.70-3.68\left(\mathrm{~m}, 1 \mathrm{H}, \mathrm{OCH}_{2}\right), 3.62-3.58(\mathrm{~m}, 1 \mathrm{H}$, $\left.\mathrm{H}-6_{\mathrm{aA}}\right), 3.56-3.51\left(\mathrm{~m}, 1 \mathrm{H}, \mathrm{CH}_{2} \mathrm{~N}_{3}\right), 3.49(\mathrm{dd}, J=8.0 \mathrm{~Hz}$ each, $\left.1 \mathrm{H}, \mathrm{H}-2_{\mathrm{B}}\right), 3.47-3.43$ (m, 1H, H- $\left.6_{\mathrm{bA}}\right), 3.42-3.37$ (m, $2 \mathrm{H}, \mathrm{H}-3_{\mathrm{A}}$, $\left.\mathrm{H}-5_{\mathrm{A}}\right), 3.25-3.19\left(\mathrm{~m}, 1 \mathrm{H}, \mathrm{CH}_{2} \mathrm{~N}_{3}\right), 1.42,1.25(2 \mathrm{~s}, 6 \mathrm{H}, 2$ $\left.\mathrm{C}\left(\mathrm{CH}_{3}\right)_{3}\right) ;{ }^{13} \mathrm{C} \mathrm{NMR}\left(125 \mathrm{MHz}, \mathrm{CDCl}_{3}\right) \delta 138.7-127.3$ (Ar-C), $109.8\left(C\left(\mathrm{CH}_{3}\right)_{2}\right), 102.5\left(\mathrm{C}-1_{\mathrm{B}}\right), 101.9\left(\mathrm{C}-1_{\mathrm{A}}\right), 80.6\left(\mathrm{C}-2_{\mathrm{B}}\right), 78.9$ $\left(\mathrm{C}-3_{\mathrm{B}}\right), 75.2\left(\mathrm{C}-5_{\mathrm{A}}\right), 74.3\left(\mathrm{PhCH}_{2}\right), 74.2\left(\mathrm{C}-3_{\mathrm{A}}\right), 74.0\left(\mathrm{C}-4_{\mathrm{B}}\right)$, $73.8\left(\mathrm{C}-2_{\mathrm{A}}\right), 73.5\left(\mathrm{PhCH}_{2}\right), 73.4\left(2 \mathrm{C}, \mathrm{C}-4_{\mathrm{A}}, \mathrm{PhCH}_{2}\right), 72.4$ $\left(\mathrm{C}-5_{\mathrm{B}}\right), 71.3\left(\mathrm{PhCH}_{2}\right), 69.4\left(\mathrm{OCH}_{2}\right), 69.3\left(\mathrm{C}-6_{\mathrm{B}}\right), 68.5\left(\mathrm{C}-6_{\mathrm{A}}\right)$, $50.8\left(\mathrm{CH}_{2} \mathrm{~N}_{3}\right), 28.2,26.4\left(\mathrm{C}\left(\mathrm{CH}_{3}\right)_{2}\right)$; MALDI-MS: $834.3[\mathrm{M}+$ $\mathrm{Na}]^{+}$; Anal. calcd for $\mathrm{C}_{45} \mathrm{H}_{53} \mathrm{~N}_{3} \mathrm{O}_{11}$ : C, 66.57; H, 6.58; found: C, 66.42; H, 6.75.

Ethyl $O$-(2,3,4,6-tetra- $O$-benzyl- $\alpha$-D-galactopyranosyl)$(1 \rightarrow 3)-4,6-O$-benzylidene-2-deoxy-2- $N$-phthalimido-1-thioB-D-glucopyranoside (9): To a solution of compound 4 (1.4 g, $2.39 \mathrm{mmol})$ and compound 5 (1.0 g, $2.26 \mathrm{mmol})$ in anhydrous $\mathrm{CH}_{2} \mathrm{Cl}_{2}-\mathrm{Et}_{2} \mathrm{O}(10 \mathrm{~mL} ; 1: 1 \mathrm{v} / \mathrm{v})$ was added MS $4 \AA$ (2.0 g), and reaction mixture was stirred at room temperature for $30 \mathrm{~min}$ under argon. The reaction mixture was cooled to $-25{ }^{\circ} \mathrm{C}$ and NIS (550.0 mg, $2.44 \mathrm{mmol})$ and $\mathrm{HClO}_{4}-\mathrm{SiO}_{2}(15.0 \mathrm{mg})$ were added. After being stirred at same temperature for $1 \mathrm{~h}$ the reaction mixture was filtered through a Celite ${ }^{\circledR}$ bed and washed with $\mathrm{CH}_{2} \mathrm{Cl}_{2}(100 \mathrm{~mL})$. The organic layer was successively washed with $5 \% \mathrm{Na}_{2} \mathrm{~S}_{2} \mathrm{O}_{3}$, saturated $\mathrm{NaHCO}_{3}$ and water, and then dried $\left(\mathrm{Na}_{2} \mathrm{SO}_{4}\right)$ and concentrated under reduced pressure to give the crude product. The crude product was purified over $\mathrm{SiO}_{2}$ by using hexane-EtOAc (7:1) as eluant to give pure compound $9(1.6 \mathrm{~g}, 74 \%)$. White solid; $\mathrm{mp} 67-68^{\circ} \mathrm{C} ;[\alpha]_{\mathrm{D}}{ }^{25}+39(\mathrm{c}$ 1.0, $\mathrm{CHCl}_{3}$ ); IR (KBr): 3417, 3063, 2870, 1774, 1715, 1610, $1495,1485,1385,1216,1099,1023,914,753,719 \mathrm{~cm}^{-1}$; ${ }^{1} \mathrm{H}$ NMR (500 MHz, $\mathrm{CDCl}_{3}$ ) $\delta$ 7.76-6.91 (m, 29H, Ar-H), 5.51 $\left(\mathrm{d}, J=3.5 \mathrm{~Hz}, 1 \mathrm{H}, \mathrm{H}-1_{\mathrm{D}}\right), 5.37$ (d, $\left.J=10.5 \mathrm{~Hz}, 1 \mathrm{H}, \mathrm{H}-1_{\mathrm{C}}\right), 5.32$ (s, $1 \mathrm{H}, \mathrm{PhCH}), 4.84$ (t, $J=9.5 \mathrm{~Hz}$ each, $1 \mathrm{H}, \mathrm{H}-3_{\mathrm{C}}$ ), 4.77-4.58 $\left(3 \mathrm{~d}, J=12.0 \mathrm{~Hz}\right.$ each, $\left.3 \mathrm{H}, \mathrm{PhCH}_{2}\right), 4.46(\mathrm{t}, J=9.5 \mathrm{~Hz}$ each, $\left.1 \mathrm{H}, \mathrm{H}-2_{\mathrm{C}}\right), 4.44$ (d, $\left.J=11.5 \mathrm{~Hz}, 1 \mathrm{H}, \mathrm{PhCH}_{2}\right), 4.34$ (d, $J=11.5$ $\left.\mathrm{Hz}, 1 \mathrm{H}, \mathrm{PhCH}_{2}\right), 4.29$ (t, $J=9.5 \mathrm{~Hz}$ each, $\left.1 \mathrm{H}, \mathrm{H}-4_{\mathrm{C}}\right), 4.19$ (d, $J$ $\left.=11.5 \mathrm{~Hz}, 1 \mathrm{H}, \mathrm{PhCH}_{2}\right), 3.86\left(\mathrm{br} \mathrm{s}, 2 \mathrm{H}, \mathrm{PhCH}_{2}\right), 3.85(\mathrm{br} \mathrm{s}, 1 \mathrm{H}$, $\left.\mathrm{H}-4_{\mathrm{D}}\right), 3.81$ (dd, $\left.J=10.5,3.0 \mathrm{~Hz}, 1 \mathrm{H}, \mathrm{H}-2_{\mathrm{D}}\right), 3.72-3.67$ (m, 3H, H-3 $\left.{ }_{\mathrm{D}}, \mathrm{H}-5_{\mathrm{C}}, \mathrm{H}-6_{\mathrm{aD}}\right), 3.58$ (br s, $\left.1 \mathrm{H}, \mathrm{H}-5_{\mathrm{D}}\right), 3.33-3.31$ (m, $1 \mathrm{H}$, H-6 $\mathrm{bD}), 3.23-3.19$ (m, 1H, H- $\left.6_{\mathrm{aC}}\right), 2.80-2.77$ (m, 1H, H- $6_{\mathrm{bC}}$ ), 2.67-2.56 (m, 2H, $\left.\mathrm{SCH}_{2} \mathrm{CH}_{3}\right), 1.12(\mathrm{t}, J=7.5 \mathrm{~Hz}$ each, $3 \mathrm{H}$, $\left.\mathrm{SCH}_{2} \mathrm{CH}_{3}\right) ;{ }^{13} \mathrm{C}$ NMR $\left(125 \mathrm{MHz}, \mathrm{CDCl}_{3}\right) \delta 168.1,167.9$ (PhthCO), 138.9-123.1 (Ar-C), 101.7 ( $\mathrm{PhCH}), 97.4\left(\mathrm{C}-1_{\mathrm{D}}\right)$,
$83.0\left(\mathrm{C}-1_{\mathrm{C}}\right), 81.7\left(\mathrm{C}-4_{\mathrm{D}}\right), 78.1\left(\mathrm{C}-3_{\mathrm{D}}\right), 75.4\left(\mathrm{C}-2_{\mathrm{D}}\right), 74.8$ $\left(\mathrm{C}-5_{\mathrm{D}}\right), 74.7\left(\mathrm{PhCH}_{2}\right), 73.3\left(2 \mathrm{C}, \mathrm{C}-3_{\mathrm{C}}, \mathrm{PhCH}_{2}\right), 72.8\left(\mathrm{PhCH}_{2}\right)$, $71.8\left(\mathrm{PhCH}_{2}\right), 70.1\left(\mathrm{C}-5_{\mathrm{C}}\right), 69.4\left(\mathrm{C}-4_{\mathrm{C}}\right), 68.8\left(\mathrm{C}-6_{\mathrm{D}}\right), 67.7$ $\left(\mathrm{C}-6_{\mathrm{C}}\right), 54.2\left(\mathrm{C}-2_{\mathrm{C}}\right), 24.0\left(\mathrm{SCH}_{2} \mathrm{CH}_{3}\right), 14.9\left(\mathrm{SCH}_{2} \mathrm{CH}_{3}\right)$; MALDI-MS: $986.3[\mathrm{M}+\mathrm{Na}]^{+}$; Anal. calcd for $\mathrm{C}_{57} \mathrm{H}_{57} \mathrm{NO}_{11} \mathrm{~S}$ : C, 71.01; H, 5.96; found: C, 70.88; H, 6.13.

2-Azidoethyl $O-(2,3,4,6-t$ etra-O-benzyl- $\alpha$-D galactopyranosyl)-(1 $\rightarrow 3)-O-(4,6-O$-benzylidene-2-deoxy-2$\mathrm{N}$-phthalimido- $\beta$-D-glucopyranosyl)-(1 $\rightarrow 2)-O$-(6-O-benzyl$3,4-O$-isopropylidene- $\beta$-D-galactopyranosyl)-( $\rightarrow 4)-2,3,6-$ tri-O-benzyl- $\beta$-D-mannopyranoside (10): To a solution of compound 8 (1.0 g, $1.23 \mathrm{mmol})$ and compound 9 (1.3 g, $1.35 \mathrm{mmol})$ in anhydrous $\mathrm{CH}_{2} \mathrm{Cl}_{2}(10 \mathrm{~mL})$ was added MS $4 \AA$ $(2.0 \mathrm{~g})$, and reaction mixture was stirred at room temperature for $30 \mathrm{~min}$ under argon. The reaction mixture was cooled to $-25{ }^{\circ} \mathrm{C}$ and NIS (350.0 $\mathrm{mg}, 1.55 \mathrm{mmol}$ ) and $\mathrm{HClO}_{4}-\mathrm{SiO}_{2}$ $(10.0 \mathrm{mg}$ ) were added to it. After being stirred at same temperature for $1 \mathrm{~h}$ the reaction mixture was filtered through a Celite ${ }^{\circledR}$ bed and washed with $\mathrm{CH}_{2} \mathrm{Cl}_{2}(100 \mathrm{~mL})$. The organic layer was successively washed with $5 \% \mathrm{Na}_{2} \mathrm{~S}_{2} \mathrm{O}_{3}$, saturated $\mathrm{NaHCO}_{3}$ and water, and then dried $\left(\mathrm{Na}_{2} \mathrm{SO}_{4}\right)$ and concentrated under reduced pressure to give the crude product. The crude product was purified over $\mathrm{SiO}_{2}$ by using hexane-EtOAc (7:1) as eluant to give pure compound $10(1.5 \mathrm{~g}, 71 \%)$. White solid; mp $65-66{ }^{\circ} \mathrm{C}$; $[\alpha]_{\mathrm{D}}{ }^{25}+34\left(c 1.0, \mathrm{CHCl}_{3}\right)$; IR (KBr): 3423, 3063, 3030, 2871, 2105, 1776, 1744, 1715, 1497, 1454, 1389, 1239, 1102, 1060, $874,737,721,697,600,530 \mathrm{~cm}^{-1} ;{ }^{1} \mathrm{H}$ NMR $(500 \mathrm{MHz}$, $\left.\mathrm{CDCl}_{3}\right) \delta 7.76-6.96(\mathrm{~m}, 49 \mathrm{H}, \mathrm{Ar}-\mathrm{H}), 5.52(\mathrm{~d}, J=3.5 \mathrm{~Hz}, 1 \mathrm{H}$, $\left.\mathrm{H}-1_{\mathrm{D}}\right), 5.42$ (d, $\left.J=8.0 \mathrm{~Hz}, 1 \mathrm{H}, \mathrm{H}-1_{\mathrm{C}}\right), 5.25$ (s, 1H, $\left.\mathrm{PhCH}\right)$, $4.86-4.45\left(\mathrm{~m}, 13 \mathrm{H}, \mathrm{PhCH}_{2}\right), 4.41\left(\mathrm{~d}, J=9.5 \mathrm{~Hz}, 1 \mathrm{H}, \mathrm{H}-1_{\mathrm{B}}\right)$, 4.37 (t, $\left.J=8.0 \mathrm{~Hz}, 1 \mathrm{H}, \mathrm{H}-2_{\mathrm{C}}\right), 4.29(\mathrm{~d}, J=11.5 \mathrm{~Hz}, 1 \mathrm{H}$, $\left.\mathrm{PhCH}_{2}\right), 4.24-4.20\left(\mathrm{~m}, 4 \mathrm{H}, \mathrm{H}-1_{\mathrm{A}}, \mathrm{H}-3_{\mathrm{C}}, \mathrm{H}-4_{\mathrm{A}}, \mathrm{OCH}_{2}\right)$, 4.13-4.08 (m, $\left.2 \mathrm{H}, \mathrm{H}-2 \mathrm{~A}, \mathrm{OCH}_{2}\right), 3.91$ (br s, $2 \mathrm{H}, \mathrm{PhCH}_{2}$ ), 3.89-3.85 (m, 3H, H-2, H-3 3 , H-3 $\left.{ }_{\mathrm{D}}\right), 3.84-3.75$ (m, 3H, H-2 $\mathrm{B}$, $\left.\mathrm{H}-4_{\mathrm{C}}, \mathrm{H}-4_{\mathrm{D}}\right), 3.70\left(\mathrm{t}, J=10.5 \mathrm{~Hz}\right.$ each, $\left.1 \mathrm{H}, \mathrm{H}-6_{\mathrm{aB}}\right), 3.64-3.60$ (m, $\left.4 \mathrm{H}, \mathrm{H}-4_{\mathrm{B}}, \mathrm{H}-5_{\mathrm{B}}, \mathrm{H}-5_{\mathrm{D}}, \mathrm{H}-6_{\mathrm{bB}}\right), 3.58-3.43$ (m, 5H, H-3 $\mathrm{A}$, H- $\left.6_{\mathrm{aA}}, \mathrm{H}-6_{\mathrm{aC}}, \mathrm{H}-6_{\mathrm{abD}}\right), 3.42-3.36$ (m, 4H, H- $5_{\mathrm{A}}, \mathrm{H}-6_{\mathrm{bA}}, \mathrm{H}-6_{\mathrm{bC}}$, $\left.\mathrm{CH}_{2} \mathrm{~N}_{3}\right), 3.34-3.26\left(\mathrm{~m}, 2 \mathrm{H}, \mathrm{H}-5_{\mathrm{C}}, \mathrm{CH}_{2} \mathrm{~N}_{3}\right), 1.27,1.25(2 \mathrm{~s}, 6 \mathrm{H}$, $\left.2 \mathrm{CH}_{3}\right) ;{ }^{13} \mathrm{C} \mathrm{NMR}\left(125 \mathrm{MHz}, \mathrm{CDCl}_{3}\right) \delta 138.6-126.3$ (Ar-C), $109.5\left(\mathrm{C}\left(\mathrm{CH}_{3}\right)_{2}\right), 101.6\left(\mathrm{C}-1_{\mathrm{B}}\right), 101.5(\mathrm{PhCH}), 100.8\left(\mathrm{C}-1_{\mathrm{C}}\right)$, $100.2\left(\mathrm{C}-1_{\mathrm{A}}\right), 97.3\left(\mathrm{C}-1_{\mathrm{D}}\right), 82.9\left(\mathrm{C}-4_{\mathrm{C}}\right), 82.7\left(\mathrm{C}-5_{\mathrm{A}}\right), 80.1$ $(\mathrm{C}-5 \mathrm{c}), 78.7\left(\mathrm{C}-3_{\mathrm{A}}\right), 78.1\left(\mathrm{C}-4_{\mathrm{D}}\right), 76.2\left(\mathrm{C}-2_{\mathrm{D}}\right), 75.5\left(\mathrm{C}-2_{\mathrm{B}}\right)$, $74.9\left(\mathrm{C}-3_{\mathrm{B}}\right), 74.8\left(\mathrm{C}-5_{\mathrm{D}}\right), 74.7\left(\mathrm{PhCH}_{2}\right), 74.6\left(\mathrm{C}-3_{\mathrm{D}}\right), 73.9$ $\left(\mathrm{PhCH}_{2}\right) 73.5\left(\mathrm{C}-3_{\mathrm{C}}\right), 73.4\left(2 \mathrm{C}, 2 \mathrm{PhCH}_{2}\right), 73.2\left(\mathrm{PhCH}_{2}\right), 72.8$ $\left(\mathrm{PhCH}_{2}\right), 72.6\left(\mathrm{C}-2_{\mathrm{A}}\right), 72.0\left(\mathrm{PhCH}_{2}\right), 71.7\left(2 \mathrm{C}, \mathrm{C}-4_{\mathrm{B}}, \mathrm{PhCH}_{2}\right)$, $69.3\left(\mathrm{C}-4_{\mathrm{A}}\right), 68.9\left(\mathrm{C}-6_{\mathrm{C}}\right), 68.8\left(\mathrm{OCH}_{2}\right), 68.7\left(\mathrm{C}-6_{\mathrm{A}}\right), 68.4$ $\left(\mathrm{OCH}_{2}\right), 67.7\left(2 \mathrm{C}, \mathrm{C}-6_{\mathrm{B}}, \mathrm{C}-6_{\mathrm{D}}\right), 65.4\left(\mathrm{C}-5_{\mathrm{B}}\right), 56.0\left(\mathrm{C}-2_{\mathrm{C}}\right), 50.8$ $\left(\mathrm{CH}_{2} \mathrm{~N}_{3}\right), 27.6,25.7\left(\mathrm{C}\left(\mathrm{CH}_{3}\right)_{2}\right)$; MALDI-MS: $1735.7[\mathrm{M}+$ $\mathrm{Na}]^{+}$; Anal. calcd for $\mathrm{C}_{100} \mathrm{H}_{104} \mathrm{~N}_{4} \mathrm{O}_{22}: \mathrm{C}, 70.08 ; \mathrm{H}, 6.12$; found: C, 69.94; H, 6.30. 
2-Aminoethyl ( $\alpha$-D-galactopyranosyl)-(1 $\rightarrow 3)$-(2-acetamido2 -deoxy- $\beta$-D-glucopyranosyl $)-(1 \rightarrow 2)-(\beta$-D-galactopyranosyl)-( $1 \rightarrow 4)-\beta$-D-mannopyranoside $(1)$ : To a solution of compound $10(500.0 \mathrm{mg}, 0.29 \mathrm{mmol})$ in $\mathrm{EtOH}(5 \mathrm{~mL})$ was added $\mathrm{NH}_{2} \mathrm{NH}_{2} \cdot \mathrm{H}_{2} \mathrm{O}(0.1 \mathrm{~mL})$ and the reaction mixture was stirred at $90{ }^{\circ} \mathrm{C}$ for $5 \mathrm{~h}$. The solvents were removed under reduced pressure, and a solution of the crude product in acetic anhydride-pyridine $(2 \mathrm{~mL}, 1: 1 \mathrm{v} / \mathrm{v})$ was kept at room temperature for $2 \mathrm{~h}$ and then concentrated. A solution of the crude product in $80 \%$ aq $\mathrm{AcOH}(10 \mathrm{~mL})$ was stirred at $80^{\circ} \mathrm{C}$ for $1.5 \mathrm{~h}$ and then concentrated. To a solution of the crude product in $\mathrm{CH}_{3} \mathrm{OH}$ $(5 \mathrm{~mL})$ were added $\mathrm{Et}_{3} \mathrm{SiH}(1.5 \mathrm{~mL}, 9.39 \mathrm{mmol})$ and $20 \%$ $\mathrm{Pd}(\mathrm{OH})_{2}-\mathrm{C}(100.0 \mathrm{mg})$ and the reaction mixture was stirred at room temperature for $6 \mathrm{~h}$. The reaction mixture was filtered through a Celite ${ }^{\circledR}$ bed and washed with $\mathrm{CH}_{3} \mathrm{OH}-\mathrm{H}_{2} \mathrm{O}(2: 1)$. The solvents were removed under reduced pressure and the product was passed through a Sephadex ${ }^{\circledR}$ LH-20 column by using $\mathrm{CH}_{3} \mathrm{OH}-\mathrm{H}_{2} \mathrm{O}$ (3:1) as eluant to furnish pure compound $\mathbf{1}$ (135.0 mg, 60\%). Glass; $[\alpha]_{\mathrm{D}}{ }^{25}+29\left(c 1.0, \mathrm{H}_{2} \mathrm{O}\right)$; IR (KBr): 3436, 2948, 1619, 1369, 1162, $669 \mathrm{~cm}^{-1}$; ${ }^{1} \mathrm{H}$ NMR (500 MHz, $\left.\mathrm{D}_{2} \mathrm{O}\right) \delta 5.31\left(\mathrm{~d}, J=8.5 \mathrm{~Hz}, 1 \mathrm{H}, \mathrm{H}-1_{\mathrm{C}}\right), 5.15(\mathrm{~d}, J=3.5 \mathrm{~Hz}, 1 \mathrm{H}$, $\left.\mathrm{H}-1_{\mathrm{D}}\right), 4.63$ (br s, $\left.1 \mathrm{H}, \mathrm{H}-1_{\mathrm{A}}\right), 4.48(\mathrm{t}, J=10.5 \mathrm{~Hz}$ each, $1 \mathrm{H}$, $\left.\mathrm{H}-3_{\mathrm{C}}\right), 4.34$ (d, $\left.J=8.5 \mathrm{~Hz}, 1 \mathrm{H}, \mathrm{H}-1_{\mathrm{B}}\right), 4.09$ (t, $J=10.0 \mathrm{~Hz}$ each, $\left.1 \mathrm{H}, \mathrm{H}-2_{\mathrm{C}}\right), 4.05-3.92\left(\mathrm{~m}, 4 \mathrm{H}, \mathrm{H}-2_{\mathrm{A}}, \mathrm{H}-4_{\mathrm{D}}, \mathrm{H}-5_{\mathrm{D}}, \mathrm{OCH}_{2 \mathrm{a}}\right)$, $3.90-3.80$ (m, 4H, H-2, H-3 $3_{\mathrm{A}}, \mathrm{H}-6_{\mathrm{aB}}, \mathrm{OCH}_{2 \mathrm{~b}}$ ), 3.78-3.57 (m, $\left.11 \mathrm{H}, \mathrm{H}-3_{\mathrm{D}}, \mathrm{H}-4_{\mathrm{B}}, \mathrm{H}-4_{\mathrm{C}}, \mathrm{H}-6_{\mathrm{abA}}, \mathrm{H}-6_{\mathrm{bB}}, \mathrm{H}-6_{\mathrm{abC}}, \mathrm{H}-6_{\mathrm{abD}}\right)$, 3.55-3.47 (m, 2H, H-2, $\left.\mathrm{H}-3_{\mathrm{B}}\right), 3.45-3.40$ (m, $2 \mathrm{H}, \mathrm{H}-5_{\mathrm{A}}$, $\left.\mathrm{H}-5_{\mathrm{B}}\right), 3.35-3.33$ (m, $\left.1 \mathrm{H}, \mathrm{H}-5_{\mathrm{C}}\right), 3.20-3.15\left(\mathrm{~m}, 2 \mathrm{H}, \mathrm{CH}_{2} \mathrm{NH}_{2}\right)$, $2.06\left(\mathrm{~s}, 3 \mathrm{H}, \mathrm{COCH}_{3}\right) ;{ }^{13} \mathrm{C}$ NMR $\left(125 \mathrm{MHz}, \mathrm{D}_{2} \mathrm{O}\right) \delta 171.5$ $\left(\mathrm{COCH}_{3}\right), 100.7\left(\mathrm{C}-1_{\mathrm{B}}\right), 100.6\left(\mathrm{C}-1_{\mathrm{B}}\right), 99.6\left(2 \mathrm{C}, \mathrm{C}-1_{\mathrm{A}}, \mathrm{C}-1_{\mathrm{C}}\right)$, $81.7\left(\mathrm{C}-3_{\mathrm{B}}\right), 79.0\left(\mathrm{C}-3_{\mathrm{C}}\right), 78.5\left(\mathrm{C}-4_{\mathrm{D}}\right), 77.0\left(\mathrm{C}-3_{\mathrm{D}}\right), 75.5$ $\left(\mathrm{C}-4_{\mathrm{A}}\right), 75.1\left(\mathrm{C}-4_{\mathrm{B}}\right), 73.5\left(\mathrm{C}-2_{\mathrm{D}}\right), 73.3\left(\mathrm{C}-5_{\mathrm{A}}\right), 71.3\left(\mathrm{C}-2_{\mathrm{A}}\right)$, $70.7\left(\mathrm{C}-4_{\mathrm{C}}\right), 70.6\left(\mathrm{C}-3_{\mathrm{A}}\right), 69.8\left(2 \mathrm{C}, \mathrm{C}-5_{\mathrm{C}}, \mathrm{C}-5_{\mathrm{D}}\right), 68.3(2 \mathrm{C}$, $\left.\mathrm{C}-2_{\mathrm{B}}, \mathrm{C}-5_{\mathrm{B}}\right), 65.5\left(\mathrm{OCH}_{2}\right), 60.6\left(\mathrm{C}-6_{\mathrm{B}}\right), 60.5\left(\mathrm{C}-6_{\mathrm{C}}\right), 60.2$ $\left(\mathrm{C}-6_{\mathrm{A}}\right), 59.2\left(\mathrm{C}-6_{\mathrm{D}}\right), 55.7\left(\mathrm{C}-2_{\mathrm{C}}\right), 39.5\left(\mathrm{CH}_{2} \mathrm{NH}_{2}\right), 23.1$ $\left(\mathrm{COCH}_{3}\right)$; MALDI-MS: $799.2[\mathrm{M}+\mathrm{Na}]^{+}$; Anal. calcd for $\mathrm{C}_{28} \mathrm{H}_{48} \mathrm{~N}_{4} \mathrm{O}_{21}$ : C, 43.30; H, 6.23; found: $\mathrm{C}, 43.14$; H, 6.45.

\section{Supporting Information}

\section{Supporting Information File 1}

1D and 2D NMR spectra of compounds $\mathbf{1}$ and 6-10.

[http://www.beilstein-journals.org/bjoc/content/ supplementary/1860-5397-8-230-S1.pdf]

\section{Acknowledgements}

A. S. thanks CSIR, New Delhi for providing a Senior Research Fellowship. This work was supported by DST, New Delhi (Project No. SR/S1/OC-83/2010).

\section{References}

1. Hisham, N. J. Trop. Pediatr. 1982, 28, 1-4.

2. Vila, J.; Gene, A.; Vargas, M.; Gascon, J.; Latorre, C.; Jimenez De Anta, M. T. J. Med. Microbiol. 1998, 47, 889-891. doi:10.1099/00222615-47-10-889

3. Kaper, J. B.; Nataro, J. P.; Mobley, H. L. T. Nat. Rev. Microbiol. 2004, 2, 123-140. doi:10.1038/nrmicro818

4. Russo, T. A.; Johnson, J. R. J. Infect. Dis. 2000, 181, 1753-1754. doi: $10.1086 / 315418$

5. Johnson, J. R. Clin. Microbiol. Rev. 1991, 4, 80-128.

6. Stenutz, R.; Weintraub, A.; Widmalm, G. FEMS Microbiol. Rev. 2006, 30, 382-403. doi:10.1111/j.1574-6976.2006.00016.x

7. Beutin, L.; Aleksic, S.; Zimmermann, S.; Gleier, K. Med. Microbiol. Immunol. 1994, 183, 13-21. doi:10.1007/BF00193627

8. Zhao, G.; Perepelov, A. V.; Senchenkova, S. N.; Shashkov, A. S.; Feng, L.; Li, X.; Knirel, Y. A.; Wang, L. Carbohydr. Res. 2007, 342, 1275-1279. doi:10.1016/j.carres.2007.03.005

9. Roy, R. Drug Discovery Today: Technol. 2004, 1, 327-336. doi:10.1016/j.ddtec.2004.10.005

10. Pozsgay, V. Curr. Top. Med. Chem. 2008, 8, 126-140. doi:10.2174/156802608783378864

11. Ada, G.; Isaacs, D. Clin. Microbiol. Infect. 2003, 9, 79-85. doi:10.1046/j.1469-0691.2003.00530.x

12. Vliegenthart, J. F. FEBS Lett. 2006, 580, 2945-2950. doi:10.1016/j.febslet.2006.03.053

13. Mukherjee, C.; Ranta, K.; Savolainen, J.; Leino, R. Eur. J. Org. Chem. 2012, 2957-2968. doi:10.1002/ejoc.201200041

14. Mandal, P. K.; Misra, A. K. Bioorg. Chem. 2010, 38, 56-61. doi:10.1016/j.bioorg.2010.01.001

15. Basu, S.; Pal, J. N. Carbohydr. Res. 1990, 208, 241-245. doi:10.1016/0008-6215(90)80103-A

16. Kihlberg, J. O.; Leigh, D. A.; Bundle, D. R. J. Org. Chem. 1990, 55, 2860-2863. doi:10.1021/jo00296a055

17. Mukhopadhyaya, B.; Collet, B.; Field, R. A. Tetrahedron Lett. 2005, 46, 5923-5925. doi:10.1016/j.tetlet.2005.06.119

18. Chakraborti, A. K.; Gulhane, R. Chem. Commun. 2003, 1896-1897. doi:10.1039/b304178f

19. Fraser-Reid, B.; Lopez, J. C. Top. Curr. Chem. 2011, 301, 1-29. doi:10.1007/128_2010_105

20. Mandal, P. K.; McMurray, J. S. J. Org. Chem. 2007, 72, 6599-6601. doi:10.1021/jo0706123

21. Madhusudan, S. K.; Agnihotri, G.; Negi, D. S.; Misra, A. K. Carbohydr. Res. 2005, 340, 1373-1377. doi:10.1016/j.carres.2005.03.007

22. Panchadhayee, R.; Misra, A. K. Synlett 2010, 1193-1196. doi:10.1055/s-0029-1219798

23. Bergonzi, M. C.; Catelani, G.; D'Andrea, F.; De Rensis, F. Carbohydr. Res. 1998, 311, 231-234. doi:10.1016/S0008-6215(98)00211-0

24. Kanie, O.; Ito, Y.; Ogawa, T. J. Am. Chem. Soc. 1994, 116, 12073-12074. doi:10.1021/ja00105a066

25. Lee, H.-H.; Schwartz, D. A.; Harris, J. F.; Carver, J. P.; Krepinsky, J. J. Can. J. Chem. 1986, 64, 1912-1918. doi:10.1139/v86-315 


\section{License and Terms}

This is an Open Access article under the terms of the Creative Commons Attribution License

(http://creativecommons.org/licenses/by/2.0), which permits unrestricted use, distribution, and reproduction in any medium, provided the original work is properly cited.

The license is subject to the Beilstein Journal of Organic Chemistry terms and conditions:

(http://www.beilstein-journals.org/bjoc)

The definitive version of this article is the electronic one which can be found at:

doi:10.3762/bjoc. 8.230 